\title{
Distinguished Reviewer Acknowledgements
}

Every year, many hard-working reviewers contribute to the high quality of the journal Abdominal Radiology. We would like to express our gratitude to those who provide exceptional and profound reviews in 2016. These individuals have been deemed to provide distinguished service to Abdominal Radiology. We very much appreciate your dedication and enthusiasm for the journal. Thank you for your commitment and the fruitful collaboration in the past year.

\author{
Ahmed Abdel Razek \\ Mukta Agrawal \\ Mahmoud Al-Hawary \\ Ersan Altun \\ Ryan Avery \\ Dennis Balfe \\ Chris Baughman \\ Deborah Baumgarten \\ Spencer Behr \\ Giulia Besutti \\ Mike Bettmann \\ Shweta Bhatt \\ Priya Bhosale \\ Candice Bolan \\ David Bolus \\ Candice Bookwalter \\ Amir Borhani \\ Andrew Bowman \\ Travis Browning \\ Mark Callaway \\ Onofrio Catalano \\ Orlando Catalano \\ Lionel Cheng \\ David Childs \\ Kedar Chintapalli \\ Joseph Collins \\ David Cosgrove \\ Abraham Dachman \\ Chandan Das \\ Anil Dasyam \\ Adrian Dawkins \\ David DiSantis \\ Kristin Dittmar \\ Nicholas Dunnick \\ Raymond Dyer \\ Eric Ehman \\ Tina Fang \\ Barak Friedman \\ Alessandro Furlan \\ Ashish Gandhe \\ Halemane Ganesh
}

Dhakshina Moorthy Ganeshan
Edmund Godfrey
Flavius Guglielmo
Mittul Gulati
Koichi Hayano
Matthew Heller
Tiffany Hennedige
Gary Horn
Simon Jackson
Tracy Jaffe
Aya Kamaya
Avinash Kambadakone
Zahra Kassam
Douglas Katz
Ravi Kaza
Philip Kenney
Ania Kielar
Myeong-Jin Kim
Young Kon Kim
Se Hyung Kim
Chan Kyo Kim
Naveen Kulkarni
Fred Lee
Sara Lewis
John Leyendecker
Guido Ligabue
Michael Lin
Christopher Lisanti
Mark Lockhart
Meghan Lubner
Antonio Luna
Andrej Lyshchik
Mladen Macanovic
Kumble Madhusudhan
Winnie Mar
Daniele Marin
Robert Marks
William Masch
Melvy Mathew
Shunro Matsumoto
Katherine Maturen
Sand

\author{
Giovanni Mauri \\ Vincent Mellnick \\ Christine Menias \\ Frank Miller \\ Brian Morse \\ Rashmi Nair \\ James Ntambi \\ Mehmet Ruhi Onur \\ Atul Padole \\ Uday Patel \\ Manuel Patino \\ Michael Patlas \\ David Paushter \\ Jason Pietryga \\ Srinivasa Prasad \\ Emilio Quaia \\ Sheng-Xiang Rao \\ Erick Remer \\ Jessica Robbins \\ Maxime Ronot \\ Andrew Rosenkrantz \\ Christopher G. Roth \\ Jurgen Runge \\ Rupan Sanyal \\ William Small \\ Farnoosh Sokhandon \\ Julie Song \\ Venkateswar Surabhi \\ Stephen Thomas \\ Jennifer Uyeda \\ Wendaline VanBuren \\ Sudhakar Venkatesh \\ Raghunandan Vikram \\ Ashish Wasnik \\ Michael Wells \\ Marcos Wolf \\ Gibran Timothy Yusuf \\ Atif Zaheer \\ Jessica Zarzour \\ Timothy Ziemlewicz
}

